\title{
A família como foco dos cuidados de enfermagem em meio hospitalar: um programa educativo
}

The family as the focus of nursing care in the hospital environment: an educational program

La familia como el foco de atención de enfermería en el ambiente hospitalario: un programa educativo

\author{
Maria Manuela Martins', Carla Sílvia Fernandes", Lucia Hisako Takase Gonçalves"I' \\ 'Escola Superior de Enfermagem do Porto, Departamento de Saúde da Pessoa Adulta e Idosa. Porto, Portugal. \\ "Universidade do Porto, Instituto de Ciências Biomédicas Abel Salazar (Doutoranda). Porto, Portugal. \\ I" Universidade Federal de Santa Catarina, Departamento de Enfermagem, Grupo de Estudos sobre Cuidados de Saude de \\ Pessoas Idosas (Membro). Florianópolis-SC, Brasil.
}

Submissão: 28-09-2010 Aprovação: 08-10-2012

\section{RESUMO}

Relato de uma experiência de programa educativo para enfermeiros sobre atenção à familia em meio hospitalar. O objetivo foi realizar capacitação de enfermeiros para a assistência da família. As estratégias de ensino/aprendizagem adotadas incidiram sobre o Modelo Calgary de Avaliação e Intervenção de Família junto a um grupo de enfermeiros de um hospital geral localizado na região norte de Portugal. Na avaliação subjetiva dos participantes, a esperiência pedagógica foi útil porque conseguiram perceber a necessidade de mudança no cuidado de enfermagem avançando para um enfoque integrado de atenção à familia. Descritores: Família; Educação em Enfermagem; Hospitais Gerais.

ABSTRACT

This paper reports an educational program experience for nurses on caring for the family, at the hospital environment. The objective was to train nurses for the care of the family. The strategies adopted for teaching/learning focused on the Calgary Model of Assessment and Family Intervention, with a group of nurses from a general hospital located in the north of Portugal. In the subjective evaluation of the participants, the pedagogic experience was useful because they could recognize the need for a change on nursing care, and move towards an integrated approach to the family attention.

Key words: Family; Education, Nursing; General Hospitals.

\section{RESUMEN}

Relato de una experiencia del programa educativo para enfermeros, sobre la atención para la familia en el medio hospitalario. El objetivo fue realizar la capacitación de enfermeros para la asistencia de la familia. Las estrategias de enseñanza/aprendizaje adoptadas incidieron sobre el Modelo Calgary de Evaluación e Intervención de la Familia, junto a un grupo de enfermeros de un hospital general localizado en la región norte de Portugal. En la evaluación subjetiva de los participantes, la experiencia pedagógica fue útil porque consiguieron percibir la necesidad de cambios en el cuidado de la enfermería, avanzando para un enfoque integrado de la atención para la familia.

Palabras clave: Familia; Educación en Enfermería; Hospitales Generales. 


\section{INTRODUÇÃO}

A evolução dos contextos histórico e sociocultural levou a família à diversidade de transformações, traduzidas em mudanças em sua estrutura, papéis e funções. Entre as novas formas de organização familiar, comumente observadas, destacam-se as famílias monoparentais e as famílias reconstituídas, que incluem adultos e crianças sem laços sanguíneos. É na família, não importa que tipo seja, que as pessoas crescem; nutrem-se fisica, psicológica e socialmente; ganham um sentido de si e de coletividade enquanto uma unidade cultural familiar; cultivam crenças e valores acerca da vida e progridem ao longo do ciclo vital, até à sua terminalidade. Também, é na família que recebem suporte material e psicológico em situações de maior estresse pessoal, pelo que, enquanto valor, a família ocupa um lugar primordial na vida dos indivíduos seja na saúde, na doença, na alegria, nas adversidades. Porém, em nosso meio hospitalar a família é tantas vezes esquecida ou ignorada, ou se é lembrada, é apenas vista como um recurso. A família exerce um papel significativo no hospital, nomeadamente na internação de adultos, idosos, crianças, mulheres grávidas. Ela deve ser parte integrante de intervenção em saúde em qualquer fase da doença e em todos os contextos de assistência, o que aponta para a relevância da relação entre o cuidado ao indivíduo e seu contexto familiar, como um factor indispensável ao cuidado integral da pessoa do paciente ${ }^{(1)}$.

A evolução do sistema de saúde colocou muitas vezes o paciente no hospital como elemento isolado e a família não integrada no processo de internação embora protagonista de negociação com o Hospital ${ }^{(2)}$. O que se observa no cotidiano hospitalar, a maior parte das vezes é, que intervenções de enfermagem são otimizadas em relação ao paciente, e a unidade familiar não é tomada por alvo do processo de cuidados $^{(3)}$. É necessário avançar e aprofundar em conhecimentos sobre família, utilizar métodos viáveis de intervenção na familia do paciente internado ${ }^{(4)}$.

Contudo, há alguns obstáculos para isso, tais $\mathrm{Como}^{(5)}$ : a) a maior parte dos enfermeiros ainda não foi exposta a ensinamentos de cuidados de família durante a sua formação, e continua a praticar enfermagem com base no paradigma de enfoque individual; b) A enfermagem tem ainda fortes traços históricos de sua prática baseada em enfoque no modelo biomédico, que centra tradicionalmente no indivíduo como cliente, e a família é vista na melhor das hipóteses como contexto e não como outro cliente; c) os enfermeiros acreditam que o estudo da família e de enfermagem de família, faz parte do senso comum não necessitando de aprendizagem e capacitação; d) falta modelos, tecnologias e instrumentos mais específicos para avaliar e intervir nas famílias.

É necessária a alteração dessa realidade porque a enfermagem tem compromisso com atenção às famílias de modo efetivo, na actualidade, no conjunto dos cuidados de saúde ${ }^{(6)}$.

Mas como alterar tal situação? É necessário investir na formação dos enfermeiros e na sua capacitação/atualização no campo profissional.

Ciente dessa problemática, foi desenvolvido junto ao departamento de formação e atualização dos recursos humanos de uma instituição hospitala, uma experiência de ensino do modelo Calgary de Avaliação e Intervenção de Família ${ }^{(7)}$. O relato e discussão dessa experiência foram acolhidos e avaliados pelo corpo de enfermeiros da instituição, e constituiu matéria do presente artigo.

\section{ENQUADRAMENTO TEÓRICO-METODOLÓGICO}

\section{É possivel a família ser o foco de cuidados de enfermagem em meio hospitalar?}

O hospital representa uma organização complexa, sua principal função consiste em dar resposta às necessidades de cuidados de saúde da população. O hospital constitui-se de setor estratégico de prestação de assitência ${ }^{(8-9)}$, trata-se de estabelecimento de elevada diferenciação, disposto de recursos tecnológicos e humanos, cujo objetivo é prestar serviços de saúde nas 24 horas do dia, desenvolvendo atividades diagnósticas, terapêuticas, cuidativas e de reabilitação em regime de internamento ou ambulatorial. Para além de oferecer cuidados à população, outras funções atribuídas, são o ensino e a investigação. Os hospitais, independente das suas possíveis insuficiências, continuam sendo locais de prática de cuidados de saúde. São os locais de ensino prático de médicos, enfermeiros e outros profissionais ${ }^{(10-11)}$. Apesar de o paciente ser referido como a figura central do hospital, a realidade revela-nos uma malha de relações e interdependências onde se movem motivações bastante diferentes. Essa realidade assume contornos bem mais complexos e menos evidentes quando se transpõe a esfera da presença da família no espaço hospitalar enquanto acompanhante e responsavel pelo seu paciente internado. Ainda em muitos dos nossos hospitais, se observam rotinas impostas por horários rígidos de visitas onde a família é tantas vezes esquecida ou ignorada ${ }^{(12)}$. As famílias ficam assim excluídas, não só do envolvimento no tratamento dos seus familiares doentes, como de atenção como cliente, em momentos estressantes por que passam pela hospitalização de seus entes queridos.

Toda mudança exige comprometimento e tempo para sua efetivação. É possivel que familias acompanhantes de pacientes internados sejam alvo de atenção da equipe hospitalar, sobretudo da enfermagem, se as considerar como parceiras no cuidado do paciente, e cliente do enfermeiro quando manifestam necessidades de saúde e de cuidados face às situações estressantes da doença e da hospitalização(6).

\section{Por que a família deve ser o foco de cuidados em ambien- te hospitalar?}

A família representa o pilar fundamental das sociedades humanas, na realidade, ela constitui a primeira base na qual a maioria dos seres humanos constrói a sua personalidade. A família constitui célula fundamental e valor inalienável da sociedade, assim reconhecida pela Declaração Universal dos Direitos do Homem que atesta a importância que a mesma assume no desenvolvimento da pessoa humana. Por isso, é essencial conceber e desenvolver um caminho para integrar a família nos cuidados de saúde das pessoas. A esse respeito, especialista e pesquisadora sobre família(5) ${ }^{(5)}$ ostula que, de forma geral: 
$\checkmark \quad$ os comportamentos saudáveis e de risco são aprendidos dentro do contexto familiar;

$\checkmark \quad$ a família é afetada quando um ou mais de seus membros têm problemas de saúde, sendo a unidade famíliar um fator importante na manutenção ou desiquilíbrio do estado de saúde e bem-estar dos indivíduos;

$\checkmark$ um dado funcionamento da família, pode afetar a saúde de seus membros, como também certos acontecimentos e comportamentos em/de cada membro, podem afetar a saúde e o equilíbrio de toda unidade famíliar;

$\checkmark$ a eficácia dos cuidados de saúde a um indivíduo se torna evidente quando se enfatiza o cuidado envolvendo a família e

$\checkmark$ a promoção, manutenção e restauração da saúde da unidade famíliar é essencial para a sobrevivência da sociedade.

A família tem sido objeto de estudo e de atenção especial nos últimos tempos em vários campos: político, social, científico, ao que a enfermagem não é alheia. A enfermagem, em todo o mundo, está se movimentando na direção da incorporacão da família nos cuidados de enfermagem em quaisquer especialidades, como também, do desenvolvimento do corpo de conhecimentos enquanto especialidade de enfermagem de família. Tal movimento tem produzido muitos marcos históricos de difusão e debates sobre o cuidado das familias ${ }^{(7)}$, sendo considerados marcos importantes:

$\checkmark \quad 1984-1^{\text {a }}$ edição de Enfermeiras e Familias de Wrigth \& Leahey;

$\checkmark 1988$ - I International Family Nursing Conference, Calgary-Canadá;

$\checkmark 1994-2^{\text {a }}$ edição de Enfermeiras e Familias de Wrigth \& Leahey;

$\checkmark \quad 1994$ - Implantação da assistência à família por meio do Programa Saúde da Família, instituído pelo Ministério da Saúde, Brasil;

$\checkmark 1995$ - Criação do Journal of Family Nursing;

$\checkmark 1997$ - IV International Family Nursing Conference, Valdivia-Chile;

$\checkmark 1999$ - International Family Nursing Research Congress, Tampere-Finlândia;

$\checkmark 2000$ - II WHO Ministerial Conference on Nursing and Midwifery in Europe, Munique;

$\checkmark 2000$ - Conferência da Ordem dos Enfermeiros de Portugal "A cada família o seu Enfermeiro";

$\checkmark 2006$ - Linhas de ação a priorizar para o desenvolvimento dos cuidados primários de Saúde (incluindo a família) em Portugal;

$\checkmark 2009$ - IX International Family Nursing Conference, m Reykjavic, Islândia;

$\checkmark 2011$ - 5 $5^{\text {a }}$ edição de Enfermeiras e Familias de Wrigth \& Leahey;

$\checkmark 2011$ - X International Family Nursing Conference, Kyoto-Japão.

O interesse pelo cuidado enfocado na família surge numa época marcada por grandes mudanças estruturais da sociedade e da família, cujas consequências para o futuro são difíceis de prever. Concretamente, em Portugal(8), os dados demográficos revelados nos últimos Censos (1991, 2001, 2011) alertam-nos para um envelhecimento da população transversal a todas as regiões do país, com aumento significativo na última década de divorciados e recasados; mais mulheres viúvas e divorciadas do que homens. Ainda, as famílias são hoje (2011) de menor dimensão com média de 2,6, o que era de 2,8 na decada anterior. As famílias de maior dimensão têm vindo a perder expressão: em 2011 as famílias com 5 ou mais pessoas representavam $6,5 \%$, em contraposição às taxas de $9,5 \%$ e $15,4 \%$ das décadas anteriores. Houve aumento de peso das famílias com 1 e 2 pessoas. As famílias unipessoais foram as que mais cresceram - 37,3\% nos ultimos dez anos. Com efeito, a dinâmica familiar se alterou de tal modo que, se era frequente constituir-se família pelo casamento e sua dissolução normalmente provocada pela morte, hoje a família tem se apresentado com novas compleições ${ }^{(8,9)}$. Dai, suas necessidades sao outras e devem ser contempladas pelos servicos sociais e de saúde se deseja-se que seus servicos tenham enfoque integral e holístico de seus usuários abrangendo as necessidades e expectativas de suas famílias.

\section{O Modelo Calgary de avaliação e intervenção de família}

O Modelo Calgary ${ }^{(4,7)}$ de avaliação e intervenção de famílias desenvolvido pelas enfermeiras pesquisadoras Wrigth e Leahey, da Universidade de Calgary, Canadá, vem sendo continuamente aperfeiçoado e divulgado nos ultimos 25 anos, desde 1984 e culminando com a $5^{\text {a }}$ edição, em 2009. Graças a sua tradução e edição em vários países, o modelo tem sido difundido e usado para o ensino em muitas partes do mundo. É abrangente incluindo a avaliação e intervenção de famílias, sendo aplicável em quaisquer contextos de prática clínica. $\mathrm{O}$ modelo, de avaliação e intervenção, fundamenta-se em um esquema conceitual necessário à compreenção do sistema familiar como uma unidade de cuidados, integrando alguns conceitos de sistemas, cibernética, comunicação, biologia do conhecimento e teoria da mudança. A primeira parte integra um sistema abrangente com trípla estrutura evolucionária e funcional de avaliação da família. Em linhas gerais, fornece duas ferramentas de avaliação estrutural : o genograma e o ecomapa. Na parte da intervenção, dá acesso a um repertório de intervenções baseadas em práticas clínicas, que ajuda os enfermeiros a manter ou alterar o funcionamento da família , no que diz respeito à cognição, comportamento e afeto.

\section{EXPERIÊNCIA DE PROGRAMA EDUCATIVO DE ENFER- MAGEM DE FAMILIA}

A experiencia de implementação de programa de enfermagem de familia para enfermeiros hospitalares aconteceu em um hospital geral situado no norte de Portugal, que atende aos servicos de Ortopedia, Cirurgia, Pediatria, Neonatologia, Obstetrícia, Ginecologia, Bloco Operatório, Consulta Externa e Urgência; com capacidade para 140 leitos; e com corpo de enfermagem composto de 200 enfermeiros. Essa experiência, 
de iniciativa de uma das autoras, enfermeira atuante nesse hospital, teve inteira aceitação por parte do corpo de enfermagem e autorização formal da direcão como da comissão de ética do Hospital. Assim todos os 200 enfermeiros que atuavam diretamente no atendimento dos clientes nos diferentes servicos referidos, e que se encontravam presentes no periodo definido da realização do programa educativo, aceitaram particpiar da experiência.

\section{Avaliação diagnóstica do contexto do ensino/aprendizagem}

O programa contou com um planejamento estratégico iniciado com um levantamento no sistema de informação de Suporte e Apoio à Prática de Enfermagem $\left(\mathrm{SAPE}^{\circledR}\right)$, de todos os Diagnósticos de Enfermagem registrados pelos enfermeiros, dos servicos de internação do hospital, por durante um mês (11/2009). O sistema SAPE ${ }^{\circledR}$ adotado pela enfermagem segue a mesma terminologia da Classificação Internacional para a Prática de Enfermagem $\left(\mathrm{CIPE}^{\circledR}\right)$, versão Beta ${ }^{(11,13)}$. Os diagnósticos constituem "decisão sobre um fenómeno que representa foco das intervenções de enfermagem".

Os diagnósticos de enfermagem registrados no sistema estavam concentrados no fenómeno de enfermagem focado no tipo Indivíduo/função (59,1\%); no tipo Individuo/pessoa/ação realizada pelo próprio $(27,2 \%)$. Diagnósticos no tipo focado no Grupo/família somaram apenas 0,7\%. Em outras palavras, os diagnósticos identificados pelos enfermeiros nos diversos contextos de cuidado, estavam, majoritariamente, associados às funções de intervenção focadas no indivíduo.

Os profissionais de saúde, não foram imunes à visão mecanicista que fragmenta os cuidados, apesar de se aceitar hoje a relação entre família e doença é, ainda, também muito evidente, o impacto limitado desta relação aplicado às intervenções de enfermagem, na qual a família é vista como contexto ${ }^{7}$. Esta fase permitiu evidenciar a essencialidade de intervir a este nível.

Tal contexto constituiu-se em estratégia motivadora para a consecução dos objetivos de ensino/aprendizagem do programa de mudanças para o enfoque de atenção à familia nos cuidados de enfermagem.

\section{Planejamento e implementação de ações pedagógicas}

O planeamento de uma intervenção é um processo metódico, e implica conhecer os fatores condicionantes onde se pretende intervir, de modo a determinar o tipo de atividades e escolher a metodologia de intervenção. A aprendizagem é um processo complexo levar o outro a aprender novas informações e mudar comportamentos pode ser deveras problemático, para ultrapassar estas dificuldades devem ser integrados fatores que aumentem a probabilidade de aprendizagem. ${ }^{16}$

Na planificação das ações pedagógicas de práticas de cuidados às Famílias em meio Hospitalar, com base nos princípios da educação de adultos, foram formulados objetivos, conteúdos, estratégias, recursos, tempo e o tipo de avaliação sempre tendo por base a aquisição de novos comportamentos e competências dos enfermeiros participantes. Os formandos de hoje são muito heterogéneos, com diferentes idades, culturas, crenças e estilos de aprendizagem. Esta variedade do grupo cria uma maior necessidade de estratégias inovadoras. ${ }^{17}$
Optou-se por diferentes métodos para colmatar esta barreira. $\mathrm{Na}$ formação dos grupos foram integrados elementos de diferentes contexto de trabalho (cirugia, ortopedia, urgência, etc), de modo a privilegiar os momentos de reflexão e partilha de experiências e reflexões.

Em todos os momentos pedagógicos os seguintes objetivos firmados estavam presentes para guiar suas ações: a) rever conhecimentos sobre desenvolvimento da família ao longo do ciclo vital; b) desenvolver capacidade de avaliação familiar e assegurar a qualidade dos cuidados prestados; c) aprofundar conhecimentos sobre desenvolvimento familiar e modelos de intervenção; d) almejar a intervenção dos profissionais de saúde no cuidado de famílias.

Como conteúdo, recursos e estratégia, incluiam-se as reflexões sobre principais conceitos de família, o modelo Calgary de avaliação e intervenção de familia e exemplos de sua aplicação, além de complementação e fixação da aprendizagem exibindo-se um filme de excertos de grandes êxitos do cinema sobre "Saúde/doença/ Família/Hospital". Por último, para síntese e avaliação da prática pedagógica adotou-se momento lúdico com o uso do Family Nursing Game um jogo de tabuleiro em que o progresso é determinado pela capacidade do jogador/equipe responder às questões sobre conteúdos de Enfermagem de Família. O jogo criado por duas das autoras, pretendeu incorporar todos os conteúdos abordados ao longo da sessão. Para jogar, cada grupo era dividido em diferentes equipas, agrupando elementos de diferentes contextos de modo a potenciar a partilha, cada equipa vai respondendo às questões do jogo divididas em seis áreas temáticas: conceitos, funções e estrutura familiar, processo familiar, enfermagem de família, avaliação na família e intervenção na família. Esta fase permitiu validar os conteúdos abordados, avaliar a aprendizagem e dirimir algumas dúvidas que pudessem ainda existir.

Para o processo de formação dos enfermeiros em enfermagem de família, recorreu-se às dinâmicas metodológicas de educação dialógica. As sessões de ensino/aprendizagem decorriam em grupos nunca superior a 16 elementos, tendo a disposição das salas sempre em configuração circular privilegiando a interação. Cada sessão durava em torno de quatro horas, e iniciava-se com espaço aberto para analisar o conceito de família e de outros temas relacionados, momento em que cada participante partilhava sua própria concepção e ao mesmo tempo, em processo dialogico, procedia-se a construção coletiva dos entendimentos dos conceitos em estudo. $\mathrm{O}$ aprendizado e aplicação prática em casos clínicos do modelo Calgary ocupou a maior parte do tempo destinado ao desenvolvimento do programa, que perdurou por um mes.

Observou-se que grande parte dos enfermeiros não conhecia qualquer modelo de avaliação de familia, nem de intervenção na família. Foram apontadas ao longo das sessões como dificuldade, o envolvimento da família, a questão do tempo. Nessa ocasião foi Ihes ensinado a modalidade "entrevista de 15 minutos" parte do modelo Calgary ${ }^{(7)}$.

\section{Avaliação da experiência educacional}

As diferentes fases do programa procuraram trazer contributos distintos e adequar-se à heterogeneidade da população 
envolvida. Na primeira fase de partilha do conceito de família foi visível uma discrepância na concepção atribuída a esse tema, nomeadamente na integração ou não de apenas laços sanguíneos. A construção coletiva desses momentos permitiu ir ao encontro dos que nos refere Wright e Leahey "a família é quem os seus membros dizem que são."(7)

A escolha de excertos de grandes êxitos do cinema procurou sensibilizar os participantes para a importância da integração da família nos cuidados de saúde, tendo-se observado um envolvimento psicológico significativo neste momento.

Os conteúdos formativos apresentados permitiram realçar a importância da utilização de um modelo de avaliação e intervenção familiar, nomeadamente um modelo claro, conciso e abrangente como é o caso do Modelo de Calgary ${ }^{(7)}$.

A apresentação de algumas estratégias de avaliação foi considerada de grande utilidade principalmente o genograma, dado que quando se considera o número de palavras a serem empregadas na descrição de fatos ali representados, ficam claras a simplicidade e a utilidade desses instrumentos ${ }^{(7)}$.

O último momento da formação com o jogo permitiu oferecer um feedback deste momento formativo e consolidar todos os conteúdos lecionados.

No âmbito geral de todo este percurso observou-me níveis de envolvimento com a família distinto de acordo com o contexto de cuidados dos enfermeiros. Os enfermeiros do serviço de urgência situavam-se num polo extremo ao observado nos enfermeiros do serviço de pediatria.

A avaliação de um projeto de intervenção pode ser observada nas mudanças produzidas, nos conhecimentos, nas atitudes e nos comportamentos da população alvo. Nesse programa tornou-se difícil prever a avaliação, dada a implicação da mudança de comportamento e adquisição de novas competências. No entanto, a avaliação dos conteudos teóricos foi validada pela aplicação positiva em casos clínicos, de respostas positivas na aplicação do jogo, além de avaliação subjetiva pessoal dos participantes conforme se lê em seus depoimentos:

Penso que minha intervenção, mesmo no bloco operatório, poderá vir a ter maior abrangência considerando a família (E104).

Quando se trabalha com crianças, a família participa nos tratamentos e temos muitos bons resultados... dai a importância de incluirmos a família com os usuários de todas as idades (E189).

É verdade que na urgência é complicado lidar com família, que está estressada. Cabe a nós enfermeiros ultrapassar estas situações (E11).

Segundo avaliação dos enfermeiros participantes, o modelo proporciona um sólido fundamento para avaliação e intervenção de família, pois é prático e de fácil aplicação.

\section{CONSIDERAÇÕES FINAIS}

A intervenção de enfermagem deve ser analisada com base numa visão holística, onde se compreenda que a doença num dos membros altera todo o equilibro familiar, pelo que os cuidados de enfermagem devem ser dirigidos às necessidades de toda a unidade famíliar e não às de um indivíduo em particular ${ }^{(14-15)}$. Entendemos o impacto da doença em todos os membros da família, assim como a influência da interação familiar sobre a sua cura.

É emergente a mudança desse contexto de cuidados, porque a família não é apenas um recurso do contexto de cuidados, mas um componente essencial na sociedade. Esta temática assume grande complexidade pelo facto de ter sido visível que a maioria profissional de saúde incidia essencialmente sobre a prática de cuidados de enfermagem individuais, assim como a maioria dos profissionais não detinha conhecimentos sobre esta temática, o que os levava a práticas baseadas nas suas próprias experiências sobre família.

Um modelo sistematizado de atenção à família como foi o caso do ensino bem sucedido do Modelo Calgary, é essencial para a formação e capacitacão, sobretudo de enfermeiros que ainda nao têm qualquer iniciação à especialidade em questão. A formação de adultos carece de um planeamento exaustivo de modo a integrar metodologias diversificadas e inovadoras para ir ao encontro das necessidades de aprendizagem dos formandos. Acreditamos que este percurso constitui uma mais valia para discussões e reflexões sobre por um a lado a formação dos enfermeiros sobre Família e por outro a evolução dos processos de ensino - aprendizagem.

\section{REFERÊNCIAS}

1. Ângelo M. O Contexto familiar. In: Duarte YA, Diogo MJ, editores. Atendimento domiciliar: um enfoque gerontológico. São Paulo: Atheneu; 2000. p. 27-31.

2. Carapinheiro G. Saberes e poderes no hospital. Porto: Edições Afrontamento; 1993.

3. Moreira I. O doente terminal em contexto familiar: uma análise da experiência de cuidar vivenciada pela família. Coimbra: Formasau; 2001.

4. Montefusco SRA, Bachion MM, Nakatani AYK. Avaliação de familias no contexto hospitalar: uma aproximação entre o modelo de Calgary e a taxonomia da Nanda. Texto \& Contexto Enferm 2008;17(1):72-80.

5. Hanson S. Enfermagem de cuidados de saúde à família teoria, prática e investigação. 2. ed. Loures: Lusociência; 2005.

6. Gonçalves LHT, Polaro SHI, Leite MT, Alvarez AM, Fernandez DLR. Atenção à familia acompanhante hospitalar do idoso internado. In: Gonçalves LHT, Tourinho FSV, 
editores. Enfermagem no cuidado de idosos hospitalizados. Barueri: Manole; 2012. p. 319-37.

7. Wright L, Leahey M. Enfermeiras e famílias: guia para avaliação e intervenção na família. 5. ed. São Paulo: Roca; 2011.

8. Ministério da Saúde (Portugual). O hospital português. Lisboa: Direcção Geral de Saúde; 1998.

9. Cardoso F. Avaliação da qualidade dos serviços de urgência: satisfação dos utentes. Porto. Dissertação [Mestrado em Enfermagem]- Instituto de Ciências Biomédicas Albel Salazar, Unieversidade do Porto; 2002.

10. Nogueira M. Necessidades da família no cuidar: papel do enfermeiro. Porto. Dissertação [Mestrado em Enfermagem]- Instituto de Ciências Biomédicas Albel Salazar, Unieversidade do Porto; 2003.

11. Barbieri FMC. A avaliação familiar pelo enfermeiro de família. Proposta de um instrumento de recolha de dados baseado em Wright e Leahey. Sinais Vitais 2004;55:23-30.
12. Silva JF. Quando a vida chega ao fim: expectativas do idoso hospitalizado e a família. Lisboa: Lusociência; 2006.

13. International Council of Nursing (ICN). Classificação internacional para a prática de enfermagem. Versão Beta 2. Lisboa: Instituto de Gestão Informática e Financeira da Saúde e Associação Portuguesa de Enfermeiros; 2000.

14. Mello R, Ichisato SMT, Marcon SS. Percepção da família quanto à doença e ao cuidado fisioterapêutico de pessoas com paralisia cerebral. Rev Bras Enferm 2012;65(1):104-9.

15. Sales CA, Schulli AP, Santos EM, Tironi NM, D'Artibale $E F$, Salci MA. Sentimento de familiares sobre futuro de um ser esquizofrênico: perspectiva para o cuidado de enfermagem. Rev Bras Enferm 2011;64(3):551-7.

16. Hayden J. Motivation and behavior change. In Deyoung S. Teaching Strategies for Nurse Educators. New Jersey: Prentice Hall; 2009. p.39-55.

17. Herrman J. Creative teaching strategies for the nurse educator. Philadelphia: Davis Company; 2008. 